\title{
The Fuzzy Multiple Correlation and Its Application to Determine the Correlation of Math Anxiety with Math Self-Efficacy and Attitude
}

\author{
Hari Purnomo Susanto*, Tatik Sutarti \\ STKIP PGRI Pacitan \\ Pacitan, Indonesia \\ *haripurnomosuanto@gmail.com
}

\author{
Agus Maman Abadi \\ Department of Mathematics \\ Universitas Negeri Yogyakarta \\ Sleman, Yogyakarta
}

\author{
Agustina Sri Hafidah \\ STKIP PGRI Pacitan \\ Pacitan, East Java, Indonesia
}

\begin{abstract}
The use of the fuzzy number is not doubtful in psychological domain. The fuzzy number provides a more real and flexible conception of psychology variable. The correlation between two variable and its form of fuzzy data can't be determined by using Pearson correlation. The correlation analysis to analyse two fuzzy correlations has been majorly introduced by some experts. Meanwhile, this article is aimed at determining multiple fuzzy correlation formula based on multiple traditional correlations. Henceforth, to demonstrate the multiple fuzzy correlation formula, the three psychological variables are involved; those are the mathematical anxiety and efficacy as well as the attitude toward mathematics. These three variables were taken from 245 respondents; students of vocational high school 11th grade. The comparison analysis between fuzzy multiple correlation and traditional multiple correlation is finally discussed in this article.
\end{abstract}

Keywords: fuzzy interval data, fuzzy correlation, math anxiety, math self-efficacy, math positive attitude

\section{INTRODUCTION}

In several studies, there had been developed for the psychological measurement tools in the fuzzy scale form [1-3]. This scale provides respondents with a fair choice in determining the answer in conformity with their choice compared to such forcing classical measuring devices. These measurements result in fuzzy data. The fuzzy data can be in the triangular fuzzy numbers, trapezium fuzzy numbers, or interval fuzzy numbers. In this article, the used data is fuzzy interval number because the interval fuzzy is able to conceptualize the people more real and flexible psychological condition compared to the classical data [4].

The correlation between the two variables having the form of fuzzy interval number cannot be analysed using available multiple correlation analysis or multiple regression. On the other hand, in solution, the correlation of each variable, with fuzzy data, can be determined using the fuzzy correlation. Some alternative formulas which can be employed are first fuzzy correlation with expected interval method [5]; second, the fuzzy correlation using centroid method [6]; third, the fuzzy correlation using fuzzy interval number $[1,7]$.

Those three aforementioned formulas can only determine the fuzzy correlation of two variables. Then, the novelty of this article is to determine the multiple correlations with the fuzzy data, the calculation of which adopts the classic multiple correlation formula and analogizes the definitions of fuzzy correlations [1,7]. The multiple fuzzy correlation is concentrated to reveal the correlation between a variable with two another variable.

To demonstrate this method analysis and compare it with the classical method, then the three psychological factors in this article having the influence toward learning mathematics are used, those are anxiety [8-10], self-efficacy [11], and attitudes [12,13].

\section{FUZZY CORRELATIONS}

\section{A. Fuzzy Instrument}

The fuzzy interval data from the following variables including mathematics anxiety, mathematics self-efficacy, and mathematics attitude. were measured using mathematic anxiety, self-efficacy, and attitude with the fuzzy scale. In this article, the fuzzy scale used is modified from [2,3]. To be more obvious, it can be viewed at [1-3] the advantages of using this instrument are two types of data could be simultaneously obtained, namely a classic and fuzzy data. Therefore, it simplifies comparison between the classic and the fuzzy method at one time. The three psychological variables were taken from 245 11th grade students from Pacitan Vocational High School 3.

\section{B. Fuzzy Number} [2]: 
A fuzzy number $x^{*}$ is a fuzzy subset of the space of real numbers, that is, a mapping $\mathbb{D}_{g^{m}} \mathbb{R} \in[0,1]$ which is normal, convex and has compact levels (i.e., the $\delta$-cut sets given by

$$
\begin{gathered}
\mathscr{D}_{0}(x)=\left\{x \in R_{x} \tilde{D}(x) \geq \delta \text { \}if } \delta \in(0,1)\right. \\
\tilde{D}_{0}(x)=\operatorname{cl}\left\{x \in R_{x} \tilde{D}(x) \geq 0\right\}
\end{gathered}
$$

$\theta_{a}(x)$ is called the $\delta$-Cut of fuzzy number $x^{*}$. Fuzzy number applications are written as finite numbers of $\delta$-Cut and written as a real number interval [2,14]. These intervals are after this referred to as fuzzy interval numbers.

\section{Fuzzy Correlation}

Then, to compare the advantages of multiple fuzzy correlations and multiple classic correlations, the data analysis was carried out by two techniques; those were, the correlation between two variables was determined by Pearson correlation and fuzzy correlation analysis [4]. The correlation of anxiety with mathematics self-efficacy and attitude was executed by using classic multiple correlation analysis, and multiple fuzzy correlations. The formula of the correlation used is as follows.

Fuzzy correlation used in this article is the extension of the Pearson correlation formula [1,7]. Fuzzy correlation is defined as follows.

Let $\left(x_{i}^{*}=\left[a_{i j}, b_{i x}, c_{i j}, d_{i}\right], Y_{i}^{*}=\left[e_{i}, f_{i x}, g_{i j}, h_{i}\right]_{x} i=1,2,3, \ldots, n\right)$ be a sequence of paired trapezoid fuzzy sample on populations $\Omega$ with its pairs of centroid $\left(c x_{0}^{*}, c y_{i}^{*}\right)$ and paris of area $\left\|x_{i}^{*}\right\|=$ area $\left(x_{i}^{*}\right)_{0}\left\|y_{i}^{*}\right\|=$ area $\left(y_{i}^{*}\right)$

$$
\begin{aligned}
& c x_{2 y}=\frac{\sum_{i=1}^{n}\left(c x_{i}^{*}-c \bar{x}^{*}\right)\left(c y_{i}^{*}-c \bar{y}^{*}\right)}{\sqrt{\sum_{i=1}^{*}\left(c x_{i}^{*}-c \bar{x}^{*}\right)^{2}} \sqrt{\sum_{i=1}^{m}\left(c y_{i}^{*}-c \bar{y}^{*}\right)^{2}}}
\end{aligned}
$$

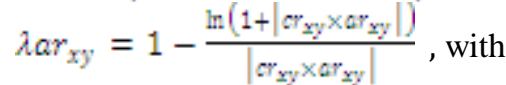

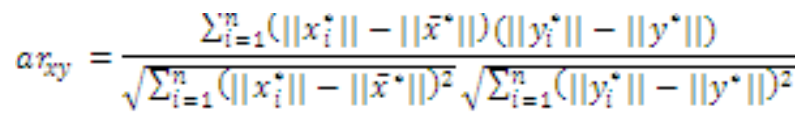

Then fuzzy coefficient correlations are defined as:

- when $c x_{2 y} \geq 0, \lambda a r_{y y} \geq 0$, The fuzzy correlation

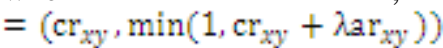

- when $c x_{y y} \geq 0, \lambda a r_{x y}<0$, The fuzzy correlation

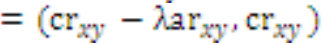

- when $c x_{y y}<0, \lambda a_{2 y} \geq 0$, The fuzzy correlation $=\left(\mathrm{cr}_{\mathrm{XY},}, \mathrm{cr}_{\mathrm{\alpha y}}+\lambda \mathrm{ar}_{\mathrm{\alpha y}}\right)$

- when $c x_{y y}<0, \lambda a_{\alpha y}<0$, The fuzzy correlation $\mathrm{cr}_{\mathrm{y}}<0, \lambda \mathrm{ar},<0$

In this article, $\operatorname{\lambda ar}_{x y}$ is used differently with [1,7]. It is carried out with assumption that value $a r_{I y y}$ is the fuzziness quality from the correlation value $C r_{I x y}$, so the value $\lambda a r_{x y y}$ is obtained above.

Multiple fuzzy relations are determined by the classic multiple correlation formula using the analogy of definition 1 .
Further, the multiple correlations are determined by the following formula

$$
\begin{aligned}
& c x_{z y}=\sqrt{\frac{c \rho_{z y}^{2}+c \rho_{z z}^{2}-c \rho_{z y} \times c \rho_{z z} \cdot c \rho_{z y}}{1-c \rho_{y z}^{2}}}
\end{aligned}
$$

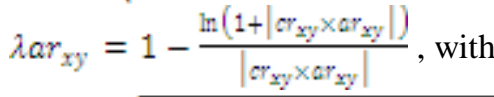

$$
\begin{aligned}
& a x_{z y}=\sqrt{\frac{\left|\rho_{x y}\right|^{2}+\left|\rho _ { z z } \| ^ { 2 } - \| \rho _ { x y } \left\|_{0}\left|\rho_{x z} \| \cdot\right| \rho_{y z} \mid\right.\right.}{1-\left|\rho_{y z}\right|^{2}}}
\end{aligned}
$$

The definition has a correlation coefficient value at intervals $[-1,1]$. Moreover, the definition is a fuzzy interval correlation $\left[x_{\text {lov }} \cdot x_{\mathrm{wg}}\right]$, where the strength of the correlation of the observed variables can be determined by the following definition.

- When $\left[x_{\text {low }}, x_{\text {wp }}\right] \in[-0.10,0.10]$, the fuzzy correlations is not significant

- When $\left[n_{\text {low }} x_{\text {wp }}\right] \in[-0.39,-0.11]$ or $[0.11,0.39]$, the fuzzy correlations is low value.

- When $\left[x_{\text {low }}, x_{\mathrm{kp}}\right] \in[-0.69,-0.40]$ or $[0.40,0.69]$, the fuzzy correlations is medium value.

- When $\left[n_{\text {long }} x_{\text {up }}\right] \in[-0.99,-0.70]$ or $[0.70,0.99]$, the fuzzy correlations is High value.

\section{EMPIRICAL STUDY}

The data description of 245 students related to anxiety, selfefficacy and students' attitudes toward mathematics with fuzzy and classic data are shown in table 1. Fuzzy data used is fuzzy interval data, and the average fuzzy interval data is determined based on the formula in the article [2,14]. The smallest limit on the fuzzy data is the smallest lower limit on the fuzzy interval data; over and above that, the biggest limit is the biggest upper limit on the fuzzy interval data $[2,4,14]$.

TABLE I. The Desciptive Of MATHEMATICS ANXIETy, SELFEFFICACY AND ATTITUDE WITH CLASSIC DATA

\begin{tabular}{|l|l|l|l|}
\hline \multicolumn{1}{|c|}{ Variable } & \multicolumn{1}{|c|}{ Average } & Minimum data & $\begin{array}{c}\text { Maximum } \\
\text { data }\end{array}$ \\
\hline Anxiety & 47.04 & 16 & 69 \\
\hline Self-Efficacy & 44.50 & 20 & 67 \\
\hline Attitude & 46.95 & 17 & 68 \\
\hline
\end{tabular}

TABLE II. ThE DESCRIPTIVE OF MATHEMATICS ANXIETy, SELFEFFICACY AND ATTITUDE WITH THE FUZZY

\begin{tabular}{|l|c|l|l|}
\hline \multicolumn{1}{|c|}{ Variable } & \multicolumn{1}{|c|}{ Average } & \multicolumn{1}{c}{$\begin{array}{c}\text { Minimum } \\
\text { data }\end{array}$} & Maximum data \\
\hline Anxiety & {$\left[\begin{array}{ll}43.25 & 53.34\end{array}\right]$} & {$\left[\begin{array}{ll}13.0 & 22.3\end{array}\right]$} & {$\left[\begin{array}{ll}67.2 & 76.4\end{array}\right]$} \\
\hline $\begin{array}{l}\text { Self- } \\
\text { Efficacy }\end{array}$ & {$\left[\begin{array}{ll}44.23 & 54.62\end{array}\right]$} & {$\left[\begin{array}{ll}12.3 & 26.8\end{array}\right]$} & {$\left[\begin{array}{ll}67.2 & 93.7\end{array}\right]$} \\
\hline Attitude & {$\left[\begin{array}{ll}47.09 & 57.14\end{array}\right]$} & {$\left[\begin{array}{ll}4.3 & 20.4\end{array}\right]$} & {$\left[\begin{array}{ll}69.3 & 86.9\end{array}\right]$} \\
\hline
\end{tabular}


As an illustration of multiple fuzzy correlations. Then, the relationship between mathematical self-efficacy and attitude together with mathematical anxiety were determined.

TABLE III. FUZZY CORRELATION COEFFICIENT

\begin{tabular}{|l|l|l|l|l|}
\hline & \multicolumn{2}{|c|}{ Fuzzy Correlation Coefficient } & \multicolumn{2}{c|}{$\begin{array}{c}\text { Classic Correlation } \\
\text { Coefficient }\end{array}$} \\
\hline Variable & Self-efficacy & Attitude & $\begin{array}{c}\text { Self- } \\
\text { efficacy }\end{array}$ & Attitude \\
\hline Anxiety & {$\left[\begin{array}{lll}-0.25 & -0.18\end{array}\right]$} & {$\left[\begin{array}{lll}-0.308 & -0.202\end{array}\right]$} & -0.251 & -0.303 \\
\hline $\begin{array}{l}\text { Self- } \\
\text { efficacy }\end{array}$ & 1 & {$\left[\begin{array}{ll}0.668 & 0.851\end{array}\right]$} & 1 & 0.674 \\
\hline
\end{tabular}

By the same token, the correlation between variables is determined by the Pearson correlation for the classic data coupled with the fuzzy correlation for the fuzzy interval data. In table 3, the fuzzy correlation and Pearson correlation fairly provide the same conception as follows: 1) mathematics anxiety with mathematics self-efficacy has a negative correlation at a low level; 2) mathematics anxiety with attitude towards mathematics has a negative correlation at a medium level; 3) the correlation between mathematics self-efficacy and attitude has a positive correlation.

Therefore, based on table 3, the multiple correlations between mathematics anxiety and self-efficacy as well as students' attitudes can be determined using the last formula. Here are the results of the multiple correlation calculation of the classic and fuzzy data, $r=0.523$ and $r=\left[\begin{array}{ll}0.514 & 0.585\end{array}\right]$ respectively. Leading to the investigation, both coefficients indicate that mathematics self-efficacy and students' attitudes have the anxiety correlation at a medium level.

\section{DISCUSSIONS}

The correlation between those variables defined by the two correlation analysis models, both classical and fuzzy fairly provides the same results related to the type and level of correlation [15-18]. Referring to the Pearson correlation analysis, both have a medium-level correlation. The results of the fuzzy correlation show that: 1) between math anxiety and math self-efficacy, they have negative correlation on the lowlevel; 2) the direction of negative correlation is also had by mathematics anxiety and students' attitudes toward mathematics [12,19,20]. This condition is equal as some previous researches, and both have a low correlation; 3) mathematics self-efficacy has a positive correlation with students' attitudes toward mathematics, the direction of this correlation is consistent with the previous research results $[19,21]$. The correlation level of mathematics self-efficacy and attitudes towards mathematics is at a medium to high-level correlation. From some of these explanations, it is found that the higher the anxiety, the lower the mathematics self-efficacy and students' attitudes towards mathematics will be and vice versa. Moreover, the higher self-efficacy of mathematics students will result in higher conditions for students' attitudes toward mathematics, and vice versa.

The multiple correlation coefficients obtained show the correlation of mathematics anxiety, self-efficacy, and students' attitudes is at a medium level. This correlation unearths that mathematics self-efficacy coupled with students' attitudes towards mathematics simultaneously influences mathematics anxiety.

The correlation coefficient in Table 3 illustrates the condition of the correlation among variables. The Person correlation coefficient provides an absolute concept, but the fuzzy correlation coefficient shows as if it seemed to be unclear or fuzzy condition. Fuzzy correlation coefficients in the form of intervals provide a flexible and real concept of the correlation between variables because the correlation among variables may have two condition correlation levels in two conditions at a time, as in the case of the correlation between mathematics anxiety and self-efficacy.

Simple and multiple fuzzy correlations provide alternative solutions in analysing students' truly complex psychological conditions [22]. Interval coefficients provide an opportunity for researchers or experts to participate in determining a single coefficient that meets each student's psychological conditions.

\section{CONCLUSION}

In conformity with the preceding discussions and findings, it can be inferred that mathematics anxiety can be reduced by increasing the mathematics self-efficacy and students' attitudes towards mathematics. Low mathematics anxiety conditions will improve student performance in carrying out tasks and learning in mathematics. It is shown by the correlation between anxiety and student self-efficacy, mathematics anxiety with students 'attitudes toward mathematics, or anxiety with a combination of mathematics self-efficacy and students attitudes toward mathematics.

Simple fuzzy correlation and multiple fuzzy correlation give results that are in accordance with human reasoning. Correlation coefficients are generated using correlation formulas for fuzzy interval data analysis, which is a flexible and real condition. Interval form of the correlation coefficient makes researchers and users more advantageous in adjusting the psychological character based on factors that did not analysed for consideration.

\section{REFERENCES}

[1] B. Wu, W.S. Sha, and J.C. Chen, "Correlation Evaluation with Fuzzy Data and its Application in the Management Science," In Econometrics of Risk, pp. 273-285, 2015.

[2] M.A. Gil, M.A. Lubiano, S.R. Saa, and B. Sinova, "Analyzing Data from Fuzzy rating scale-based Questionnaire. A Case Study," Psicothema, vol. 27, pp. 182-191, 2015.

[3] M.A. Gil and G. González-Rodríguez, "Fuzzy vs. Likert scale in statistics," In Combining experimentation and theory, pp. 407-420, 2012

[4] T. Sutarti, H.P. Susanto, A.S. Hafidah, M. Abdul, and M. Anwar "Evaluating Anxiety, Self-Efficacy and Positive Attitude Correlation Towards Mathematics Through Fuzzy Correlation Utilization," Journa of Engineering and Applied Sciences, vol. 14, no. 7, pp. 2340-23441, 2019.

[5] W.L. Hung and J.W. Wu, "A Note On The Correlation Of Fuzzy Numbers By Expected Interval," International Journal of Uncertainty, Fuzzyness and Knowledge-Based Systems, vol. 9, no. 4, pp. 517-523, 2001 . 
[6] I.M. Hanafy, A.A. Salama, and K.M. Mahfouz, "Correlation Coefficients of Neutrosophic Sets by Centroid Method," International Journal of Probability and Statistics, vol. 2, no. 1, pp. 9-12, 2013.

[7] Y. Cheng and C.C. Yang, "The application of fuzzy coefficient correlation with fuzzy interval data," International Journal of Innovative Management, Information \& Production, vol. 5, no. 3, pp. 67-68, 2014.

[8] M.H. Ashcraft, "Math anxiety: Personal, educational, and cognitive consequences," Directions in Psychological Science, vol. 11, pp. 181$185,2002$.

[9] R.K. Vukovic, M.J. Kieffer, S.P. Bailey, and R.R. Harari, "Mathematics anxiety in young children: Concurrent and longitudinal associations with mathematical performance," Contemporary educational psychology, vol. 38, no. 1, pp. 1-10, 2013.

[10] G.J. Zhan, N. Zhao, and Qi Ping Kong, "The Correlation Between Math Anxiety and Math Performance: A Meta-Analytic Investigation," Frontiers in Psychology, vol. 10, 2019.

[11] A. Kundu and A. Ghose, "The Correlation Between Attitude And Self Efficacy In Mathematics Among Higher Secondary Students," IOSR Journal of Humanities and Social Science, vol. 21, no. 4, pp. 25-31, 2016.

[12] M. Chaman and R. Callingham, "Correlation Between Mathematics Anxiety and Attitude Towards Mathematics Among Indian Students," Proceeding of the 36th annual conference of the mathematics Educations Research Group of Australia. Melbourne, 2013, p. 283.

[13] R. Zan and P.D. Martino, "Attitudes toward Mathematics: Overcoming positive/negative dichotomy," The Montana Mathematics Enthusiasts Monograph, vol. 3, pp. 157-168, 2007.

[14] R. Viertl, Statistical methods for Fuzzy data. Aptara Inc (New Delhi, India), 2010, pp 23-25.
[15] F.S. Azar and L. Mahmoudi, "Relationship between Mathematics, selfefficacy and students' performance in statistics: the meditational role of attitude toward Mathematics and Mathematics anxiety," Journal of Educational Sciences \& Psychology, vol. 4, no. 1, 2014.

[16] B. Hoffman, "I think I can, but I'm afraid to try: the role of self-efficacy beliefs and mathematics anxiety in mathematics problem-solving efficiency," Learning and Individual Differences, vol. 20, pp. 276-283, 2010.

[17] J. Lee, "Universals and specifics of math self-concept, math selfefficacy, and math anxiety across 41 PISA 2003 participating countries," Learning and Individual Differences, vol. 19, pp. 355-365, 2009.

[18] A.L. McGrath, A. Ferns, L. Greiner, W. Wanamaker, and S. Brown, "Reducing Anxiety and Increasing Self-efficacy within an Advanced Graduate Psychology Statistics Course," The Canadian Journal for the Scholarship of Teaching and Learning, vol. 6, no. 1, pp. 1-17, 2015.

[19] A. Akin and I.N. Kurbanoglu, "The Correlation Between Math Anxiety, Math Attitudes, And Self-Efficacy: A Structural Equation Model," Studia Psychologica, vol. 53, vol. 3, pp. 263-273, 2011.

[20] R. Sanci, Correlation among math anxiety, attitudes toward math, and math achievement in grade 9 students: correlation across gender.Disertation. Faculty of Education, Brock University St. Catharines, Ontario, 2014.

[21] S. Recber, M. Isiksal, and Y. Koç, "Investigating Self-efficacy, Anxiety, Attitudes and Mathematics Achievement Regarding Gender and School type,” Anales De Psicología, vol. 34, no. 1, pp. 41-51, 2018.

[22] M. Lalla and G. Facchinetti, Measurement and fuzzy scales Misurazione e scale sfocate [Online]. Retrieved from: https://www.semanticscholar.org /paper/Measurement-and-fuzzy-scales1-Misurazione-e-scaleLalla/97f2ac58ad0bb09a9ac87ead82228c22c6b0b261, 2004. 